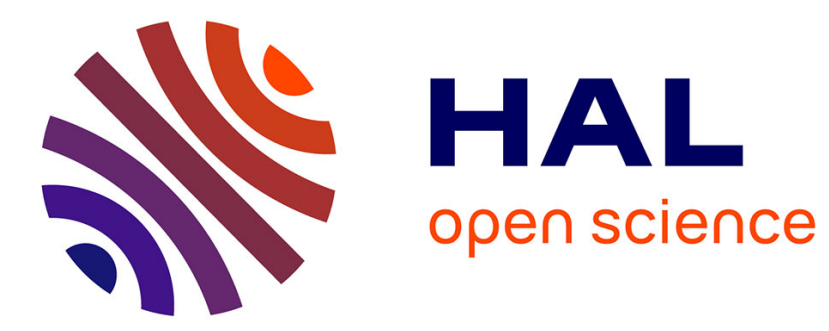

\title{
Vivre par le sport, ou comment devenir soi
}

Florian Houssier

\section{To cite this version:}

Florian Houssier. Vivre par le sport, ou comment devenir soi. Adolescence, 2014, 32, pp.259 - 272. 10.3917/ado.088.0259. hal-01423195

\section{HAL Id: hal-01423195 \\ https://hal.science/hal-01423195}

Submitted on 7 Jan 2017

HAL is a multi-disciplinary open access archive for the deposit and dissemination of scientific research documents, whether they are published or not. The documents may come from teaching and research institutions in France or abroad, or from public or private research centers.
L'archive ouverte pluridisciplinaire HAL, est destinée au dépôt et à la diffusion de documents scientifiques de niveau recherche, publiés ou non, émanant des établissements d'enseignement et de recherche français ou étrangers, des laboratoires publics ou privés. 


\section{$\underline{\text { Vivre par le sport, ou comment devenir soi }}$}

\section{Florian Houssier}

Article paru dans Adolescence, 32, 2, 2014, p. 259-272.

Résumé : Entre fétichisation du corps devenu un repère de la valeur du sujet et maintien du jeu comme modalité d'obtention de satisfactions narcissiques et libidinales, le sport est devenu une source d'investissement à la croisée du sujet, du social et du politique. A partir de la biographie d'André Agassi, célèbre joueur de tennis des années 1990, nous explorons les divers aspects de l'emprise parentale - ici un père - sur le destin psychique d'un sportif de haut niveau.

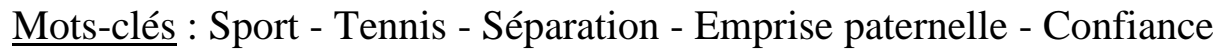

Depuis toujours, le sport est un mobilisateur du lien social, comme il en révèle ses apories. L'origine étymologique de ce terme anglais renvoie au «desport », à l'amusement. Des jeux du cirque aux jeux olympiques, des sphères politiques à celles des finances, le sport relève d'une articulation entre corps social et mise en acte pulsionnelle du corps subjectal. Entre la proie et l'ombre, la professionnalisation des sports les plus populaires a transformé progressivement une modalité d'expression individuelle et/ou groupale en métier; la performance en est devenue une obligation, oblitérant souvent les aspects les plus ludiques que les amateurs recherchent, encore et encore. Le corps libidinalisé, largement exploré dans ce numéro, rencontre le déploiement du corps pubère en même temps qu'un choix de vie : entrer, souvent trop tôt, dans un investissement quasi-fétichisé d'un corps devenu un repère de la valeur du sujet, ou diffracter les investissements et maintenir le jeu comme modalité d'obtention de satisfactions narcissiques et libidinales. Ce dernier aspect est particulièrement présent dans les manifestations sportives affichant désormais le sport comme une pause récréative et collective ${ }^{1}$.

M. Klein avait déjà remarqué que le sport permettait de passer par le corps pour détruire en fantasme le mauvais objet in-corporé, ouvrant la voie de la proposition winnicottienne selon laquelle, à l'adolescence, grandir est un acte agressif. Or, le sport est un domaine de prédilection des adolescents, formalisés dès l'enfance par les liens identificatoires aux

\footnotetext{
${ }^{1}$ On pense ici à des courses à pied en forêt avec des obstacles dits « funs », comme la session annuelle de «Got Balls! », répondant à cet accordage entre affirmation phallique et suspension relative de toute notion de compétitivité.
} 
investissements culturels parentaux et sociaux. Comment ne pas penser à la façon dont certains garçons s'identifient à des figures footballistiques appréciées par leur père, figures participant de moments de partages d'affects régulièrement mobilisés ? En ces temps de coupe du monde au Brésil, voilà un sport qui offre une caisse de résonnance identificatoire à effets multiples, articulant idolâtrie individuelle, nationale et évènement mondial.

Dans la culture, l'histoire de sportifs a fait l'objet de biographie, de films ou de romans, tant elles sont contrastées, parfois jusqu'au drame. Un exemple? Dans les années 1990, le joueur de tennis argentin G. Coria arrive en finale de Roland Garros, le Graal des joueurs spécialistes de la terre battue. Il obtient deux balles de match, mais perd contre un de ses compatriotes, moins talentueux. Il ne s'en remettra jamais. A la suite d'un pic dépressif accompagné de blessures à répétitions, il tente un come-back en pure perte : il lui devient impossible de servir sans faire de doubles fautes, empêchant ainsi tout retour au plus haut niveau. Apres avoir consulté un psychologue, faute de dépassement de cette inhibition massive, il arrêtera sa carrière avec sans doute d'immenses regrets face à tant d'insistance auto-punitive. La langue de chaque sport est porteuse de signifiants parfois peu énigmatiques : "faire une double faute », ou « la mettre au fond », «ça » parle tout seul.

Des blocages psychologiques entravant un talent pourtant singulier, des potientialités physiques en tension, entre blessures et toute-puissance, un lien d'emprise, souvent avec les pères, autant de thématiques qui touchent l'adolescence et seront explorées dans ce numéro comme dans cet article. Et pourtant, comment ne pas évoquer le corps adolescent comme celui qui, même si la détection de talents est devenue de plus en plus précoce, révèle un quelque chose en plus, comme aller plus haut, être (le) plus fort, avec en point de mire une professionnalisation dont on pressent les risques et autres chausse-trappes ? Une séparation aussi précoce que brutale avec l'environnement familial pour rejoindre un centre de formation, l'espoir des parents contrastant avec le désespoir de l'adolescent; la pression constante des formateurs sportifs, qui poussent sans cesse à devenir plus performant, les difficultés à trouver sa place dans un groupe, ou encore une fin de carrière avant qu'elle ait vraiment commencée, sans compter les retraites qui laissent le jeune adulte exsangue, sans but ni désir ; autant de thématiques qui s'articulent avec un des conflits centraux de l'adolescence de ces sportifs : comment se construire, comment devenir soi quand son désir est aussi emmêlé avec celui des autres, la famille, l'entourage, la presse, le pays d'appartenance ? Comment s'approprier un sentiment d'appartenance subjectivant quand, de l'intérieur comme de l'extérieur, le désir vient de l'autre au point de s'en sentir dessaisi ? Comme on parle parfois de vol d'enfance, on peut parler ici de vol subjectal à l'adolescence. 


\section{L'adolescent sportif, entre séparation précoce et addiction}

L'adolescent a treize ans, il est doué pour le football, il a été repéré pour jouer dans l'équipe de jeunes de sa ville ; une quinzaine d'années plus tard, il racontera ses souvenirs dans le journal L'Equipe: ses parents sont convoqués par le club, et ils rencontrent la personne chargée de la formation des jeunes, qui leur tient ce propos : soit votre fils reste ici et il restera un joueur qui s'amuse, soit vous acceptez qu'il parte (à environ trois cent kilomètres de là) dans un club formateur et il a le potentiel pour gagner sa vie en devenant professionnel. Lui sait de quoi il retourne, et il attend dans le couloir, se demandant ce que ses parents vont choisir pour lui ; il se dit qu'il resterait bien dans sa famille, que ce n'est pas grave s'il ne devient pas footballeur, même si c'est un bien joli rêve charriant son lot de fantasmes héroïques. Les parents sortent du bureau, et lui annoncent qu'ils ont été convaincus par le formateur, il partira bientôt. Soutenu par ses parents, il deviendra un joueur professionnel dans une équipe de Ligue 1, mais le rêve a ses revers : aujourd'hui marié avec deux enfants, il évoque une troublante anecdote; lorsque sa mère et lui parlent de son fils, une boule d'émotion le submerge, ils s'approchent de la surface de réparation, rarement évoquée : la séparation trop tôt venue entre mère et fils se rejoue à travers les remarques de la mère sur la façon d'éduquer son fils, elle est à la limite du hors-jeu, un jeu qu'ils partagent dans le non-dit depuis toutes ces années. La décision des parents est restée comme une souffrance mal cicatrisée, sous forme d'une séparation traumatique, et lorsqu'il est question de son fils, il commence des phrases sans les terminer : «Oui mais avec moi, vous...». Tous deux savent de quoi il retourne, tous deux sont émus et préfèrent, pour que le vent ne devienne pas tempête, rester sur le suspens, le sous-entendu partagé.

Regardons ailleurs un instant ; quand un journaliste demande à Kobe Bryant, un des meilleurs basketteurs actuels, ce qu'il pourrait faire après sa retraite de joueur, celui-ci répond qu'il ne pourra pas trouver de palliatif au basket. Pallier les sensations fortes, l'adrénaline qui devient une sorte de substance toxique endogène poussant à chercher sans cesse à retrouver un corps érotisé comme source de sensations libidinales uniques, inouïes, rendant le monde externe sans goût ni saveur. Freud (1905) avait proposé un rapprochement entre satisfaction sexuelle et dépendance, considérant que la première source de toxicomanie était une chimie d'essence sexuelle. La dépendance à l'objet corps apparait désormais, au moi corporel auraient pu écrire Freud et Paul Federn à sa suite, dépendance qui ne saurait être élaborée per sui. 
Freud et Federn, parlons-en. Federn fut un pionnier pour interroger la question du nationalisme, notion désormais si prégnante dans ce que mobilise le sport ; il était le bras droit de Freud à Vienne. On a souvent considéré que Freud était un homme d'une seule pièce, qui avait surmonté héroïquement non seulement son addiction à la cocaïne mais surtout ses résistances, internes comme externes, pour mieux accoucher la psychanalyse. Tout indique en effet que Freud a renoncé, au tournant du siècle, à toucher le corps de ses patientes ainsi qu'à la suggestion hypnotique. Mais ce serait une trop courte vue de considérer qu'il n'existe qu'un seul Freud, et que le rapport au corps a progressivement disparu sous les effets de son autoanalyse et du supposé abandon de sa théorie de la Neurotica. Pour reprendre sa propre formule, il n'a pas renoncé, il a fait du troc. Ainsi, quand il s'adresse à Federn pour lui adresser des patients qu'il ne veut ou peut plus recevoir, un autre Freud apparait, plus pragmatique et incluant toutes sortes de psychothérapies. Comme il y aurait aujourd'hui plusieurs psychanalyses, il y a «plusieurs Freud». Ses prescriptions en tant que consultant montrent que ce Freud là était sans doute le premier psychosomaticien avant l'heure (Houssier et al, 2014). La lettre qu'il adresse à Federn le 5 mai 1919 est significative : «Après avoir observé les convulsions de la patiente ${ }^{2}$, j'aimerais vous faire deux propositions pour la thérapie. Premièrement des massages superficiels locaux (par effleurage) et deuxièmement un endormissement par hypnose (sans suggestions thérapeutiques directes). Pour commencer, une semaine de repos au lit. Salutations confraternelles. Freud ». Massage, sommeil et hypnose sont intégrés sans contradiction dans la prise en charge de cette patiente. On ne saurait mieux associer de telles propositions à ce que tout encadrement sportif induit dans le mode de vie d'un sportif de haut niveau.

\section{« Je ne fais que ce qu'on attend de moi » (Agassi, 2009, p. 49)}

La biographie d'André Agassi (2009), joueur de tennis fantasque des années 90, est d'une qualité rare et fait déjà référence ; loin des biographies aseptisées, le joueur retraité s'y livre sans fard, avec une sincérité désarmante. Ses insights, le récit de son histoire infanto-juvénile, illustrent avec acuité la conflictualité à la fois typique et particulière d'une adolescence dominée par le rapport au sport de haut niveau.

Lorsqu'il fait face à ce qui pourrait être son dernier match officiel, il se sent sur le point de mourir, pas prêt, toujours isolé et en train de se parler à lui-même, comme en réclusion sur

\footnotetext{
${ }^{2}$ Dans le sens hystérique ici.
} 
une île, ajoute-t-il. Cassé de partout - surtout du dos -, Agassi livre une première impression très personnelle : il hait le tennis depuis son plus jeune âge, du moins le croit-il profondément. Certainement hait-il celui qui lui a fait aimer et détester le sport qui a fait sa fortune et sa célébrité, son père, immigré américain d'origine iranienne. Obsédé par la réussite sportive qui l'avait fui en tant que boxeur, le pater familias voulait qu'un de ses enfants devienne champion de tennis, tandis que sa mère imaginait son fils André prédicateur ; contrairement à son frère aîné qui s'y essaya et sa sœur qui se maria avec un ancien champion, André avait un don naturel.

Naturel ? A voir. Conditionné par un père vindicatif, celui-ci, dès avant sa naissance, rêvait d'un fils champion; il interprète le seul de mouvement des yeux de son fils d'un an regardant une partie de ping-pong comme le signe d'un don technique inné. Au-dessus du berceau, le mobile sera fait de balles de tennis. A trois ans, le petit André avait l'autorisation de son père de frapper ce qu'il voulait avec une raquette à manche (à canon ?) scié ; les salières traversent les fenêtres, le chien est battu, rappelant que le père a été battu par sa mère. Il joue avec une balle dès deux ans, avant d'affronter une machine sur un court de tennis, qu'il surnommera « le dragon » et crache à un rythme effréné des balles jaillissant de sa gueule toujours ouverte. Au même moment, son père lui hurle : «Frappe plus tôt !», injonction interchangeable avec «Frappe plus fort ! ». Difficile de ne pas penser à ce talent précoce que fut Agassi, chargé, à la suite de la mission paternelle puis nationale, de représenter son pays en tant que nouveau sauveteur du tennis américain.

Ce déplacement de la figure paternelle tyrannique au jeu lui-même ne résulte pas tant d'un syndrome d'influence, ou d'un trop clivant syndrome de réussite par procuration, mais plutôt d'un CEdipe négatif renforcé par la passivité de l'attitude maternelle et de l'empiètement précoce sur la psyché du petit André, mandaté inconsciemment par son père pour réparer sa dépression. Si l'expression de sa destructivité a été favorisée, Agassi semble avoir été attaqué dans sa capacité à éprouver le plaisir de jouer, enchevêtré dans un conflit insoluble : jouer avec plaisir revient à céder au tyran, jouer en haïssant le tennis complique la possibilité de gagner. Et les défaites jalonnent le parcours tennistique, pourtant brillant, de ce talent du tennis mondial que fut A. Agassi. Ces deux parts de lui en conflits tout au long de sa carrière feront penser à certains qu'il souffre de troubles bipolaires, alors qu'il est avant tout question de trouver son désir pour ne pas dépérir, un sentiment d'identité suffisamment stable pour avoir l'impression d'un choix possible.

Il ne fut pas seulement sous l'emprise paternelle ; parfois contraint intérieurement, pour ne pas le blesser, de cacher ses résultats à son frère aîné qui fut également l'enfant dépôt de 
l'ambition paternelle, Agassi montre dans sa biographie le parcours infanto-juvénile de quelqu'un qui cherche sans cesse comment exister pour soi et non seulement dans le désir de son père et de sa famille. «Je ne fais que ce qu'on attend de moi », écrit-il, en ajoutant : «Chaque coup réussi est normal, chaque coup raté provoque une crise », celle de son père qui, intériorisé, deviendra la sienne. Seule la sensation de frapper un coup vécu comme absolument parfait lui permet de ressentir une fugace impression de calme, de bien-être et de paix intérieure, mais non de joie ou de plaisir.

Lorsque l'école arrive dans sa vie, il faut lui répéter plusieurs fois les choses pour qu'il les comprenne; non parce qu'il est moins intelligent que les autres, mais parce que son père prend toute la place dans sa tête. Agassi associe sa difficulté à l'identification au désir introjecté et excorporé de son père en lui : son père lui hurlait toujours les choses deux fois, considérant que penser est contraire au jeu en action. Deux interdits, implicite et explicite, impriment leur marque : rêvasser et penser sont proscrits, ces activités psychiques s'opposant à l'action. Son hypermaturité maniaque, visible y compris sur le court, trouve là une de ses sources internes. L'école fut également le lieu de l'humiliation du père lorsque, en Iran, sa mère lui imposait parfois d'aller à l'école habillé en fille, soumis ainsi à la moquerie de tous et contraint d'apprendre à se battre. Le père découvre le tennis avec des figures identificatoires masculines, auprès de GIs à qui il rendait des services. En l'absence de tout dialogue possible avec d'autres enfants jouant au tennis, il deviendra boxeur avant de s'exiler aux Etats-Unis pour fuir l'Iran.

Lorsque, à leur plus grande joie, le père propose à ses enfants de faire l'école buissonnière pour aller «taper des balles », il court-circuite la position de la mère : «Pas un mot à votre mère », ajoute-t-il (Agassi, Ibid, p. 58), alimentant une complicité incestuelle toute à son profit de séducteur narcissique. Enfermé par son père dans un court de tennis ou en voiture avec lui, enfermé intérieurement par une série de contraintes imposées par hurlement, Agassi écrit : «J'ai intériorisé mon père, son impatience, son perfectionnisme, sa rage, au point que sa voix n'est plus très différente de la mienne (...). Je n'ai plus besoin que mon père me torture. A partir de ce jour, je vais m'en charger moi-même. » (Agassi, Ibid, p. 66). Grâce au conseil de son frère aîné, il pourra cependant refuser par la ruse le «speed », une petite pilule blanche que son père veut lui faire avaler pour améliorer ses performances en match. Il joue alors à un autre jeu : il perd volontairement sous les yeux de son père, ajoutant en sortant du court qu'il ne sent pas bien, ce qui découragera le père de lui proposer à nouveau un produit dopant. 


\section{Frapper plus fort pour mieux battre son père}

$\mathrm{Au}$ moment où, encore enfant, il est sur le point de battre son père au tennis, le père quitte brusquement le court et ne jouera plus de match avec son fils ; défaite hémorragique pour le père, victoire essentielle pour le fils : «(..) la plus belle victoire de ma vie », celle «qui obligera mon père à s'éloigner de moi » (Ibid, p. 88). Le roman familial du jeune André est alors d'être adopté, ou de trouver un entraîneur qui ne sera pas seulement un père bis, mais un être humain qui le comprendra, le guidera et l'aidera à sortir de la confusion des psychés. En attendant cet entraîneur aux contours d'un père plus compréhensif, moins furieux, les amitiés prennent un tour paternalisé : être un père suffisamment bon l'un pour l'autre, voilà le pacte que scellent Agassi et son ami d'adolescence qui s'épanchent sur leur père respectif.

Puis le père d'André prend conscience qu'il existe une limite à son désir : au vu des progrès de son fils devenu adolescent, il ne peut plus le former, il est déjà dépassé techniquement et peut le repérer. Il décide alors de façon unilatérale d'envoyer son fils chez Nick Bollettieri, un ancien parachutiste reconverti en coach formant des jeunes au coup droit dévastateur. Abandonné dans ce qu'il vit comme un camp de prisonniers formés de super-héros adolescents, la pression est constante : peu de contacts avec le monde extérieur, hypercompétitivité, absence de présence d'adultes, une atmosphère de jungle sur fond de violences diverses s'instaure. L'école, en dehors de l'anglais et de la poésie, est l'objet d'un désinvestissement renforcé; à quatorze ans, il obtient d'en être dispensé pour mieux se consacrer à son talent sportif. Heureusement, il reste à André sa révolte, une rébellion passant par l'apparence : piercing, boucle d'oreille, cheveux à l'iroquoise teintés en rose, puis plus tard short en jean, le corps et ses atours parlent pour lui. Cette situation n'est pas sans rappeler le propos de Meltzer (1989) : à défaut de pouvoir acquérir ce que le supposé monde adulte a obtenu, les adolescents se rabattent sur l'esthétique de l'apparence. Cet investissement d'une esthétique même opposée aux critères ambiants se déplierait au détriment de la reconnaissance de la vie psychique, laissant place à une forme de «génie cynique »; cet état d'esprit, constate-t-il, domine dans la mentalité de la bande et il «est dans son essence délinquant » (Ibid, p. 250).

Comme Agassi est un élève surdoué, on ne peut pas lâcher la future poule aux œufs d'or. Alors on lui passe ses actes de vandalisme ou ses propos injurieux envers Bollitieri, ce père substitutif de pacotille qui a des dollars dans les yeux lorsqu'il regarde le jeune prodige. Sur le court comme en dehors, André continue de dialoguer symboliquement avec son père, en le provoquant sur son homosexualité potentielle. André, lui, sait que la question n'est pas tant là 
que de se venger de ce que son père lui a fait subir, et qu'il commence à retourner activement à son avantage ; après tout, ne reprend-il pas à son compte l'histoire de son père forcé d'aller en cours habillé en fille? Cette fois, André a le choix des armes : s'identifier au père en le pastichant, en lui rappelant la brulure narcissique d'une ancienne humiliation. Cette fois, c'est lui qui choisit au lieu de subir les choix paternels. Mais il y a aussi cela : en s'opposant, il s'érige contre, tout contre son père. Cette érection, «(...) violente, involontaire, irrépressible » est acceptée comme «les nombreux changements que connaît mon corps », écrit-il (Agassi, op. cit., p. 133). Se montrer en toute exubérance est une façon de se dissimuler, de cacher sa sensibilité tout en exprimant sa colère, son sentiment d'être différent. Le lien infantile à son père rencontre les effets psychiques de l'adolescence, alors que le corps est déjà surinvesti. A seize ans, il dresse cet implacable constat : il est entré dans les cent meilleurs joueurs du monde, mais il est associé à un entraîneur montré du doigt comme un escroc et se trouve sans amis ni petite amie. Le prix de la conquête désirée par le père lui semble alors exorbitant. Tout en commençant à gagner des tournois, à sourire à une belle fille qui lui tend un gros chèque, il constate : «Je suis un adolescent qui en a déjà trop vu » (Ibid, p. 180), un homme-enfant ajoute-t-il, qui ressent le besoin de constituer une équipe autour de lui, des gens qui « organisent une sorte d'éducation permanente » (Ibid, p. 225).

Ce qu'il vit comme une libération reviendra plus tard sous la forme de la création d'une académie formant les enfants et adolescents à suivre leur scolarité dans un environnement favorable. Lorsque Bollitieri l'abandonne sans le lui en parler directement, il ressent l'envie d'aller jouer : «J'ai besoin de la douleur que seul le tennis peut procurer» (Ibid, p. 280). Douleur masochique et violence mêlées, il utilise son corps comme le vecteur de pulsions qui sont des figures-fond de son monde interne, sur lesquelles il sait pouvoir s'appuyer: s'épuiser, cogner, autant de décharges libidinales et agressives auto-calmantes.

\section{Devenir le numéro 1}

Sur le court, un conflit entre son envie de gagner et son désir d'opposition au père fait rage : arrivé deux fois en finale de grand chelem, il triomphe à sa troisième tentative. La question de l'échec devant la réussite se pose : échouer masochiquement, en souffrant passivement, devient un choix possible pour s'opposer à la voix intériorisée de son père, en nourrissant l'illusion que c'est un choix personnel, même inconscient, alors qu'il se contente d'être contre son père. Cette forme d'opposition s'accompagne d'un paradoxe: libérer ses pulsions nécessite une maitrise tandis que perdre en jouant mal relève d'une inhibition auto-punitive 
sans doute plus facile à atteindre. Le sentiment d'imposture qui en découle s'articule avec l'omniprésence de la voix paternelle: gagner pour son père est une forme d'imposture. Lorsqu'il devient numéro un mondial, il aimerait ressentir de la joie ou de la fierté, au lieu du sentiment de vide et d'absence d'envie qui le taraudent. Prendre des méta-amphétamines pour se débarrasser da la vague de tristesse qui l'envahit provoquera même un contrôle antidopage positif, dont il se sortira grâce à une bien suspecte bienveillance de l'association internationale de tennis, sans doute peu désireuse de détruire l'image d'un champion et de salir celle du tennis. Entre dépression et confusion, sa carrière sera jalonnée de hauts et de bas, entre des blessures et des moments de grâce où il survole le circuit. La mise en cause de son perfectionnisme l'aide à lâcher, à ressentir moins de pression interne quant à l'obligation de gagner avec panache ; lorsqu'il fait appel à un entraîneur qui vient d'écrire un livre titré ironiquement «Winning Ugly»(Gilbert, 1994), soit gagner en jouant affreusement, il ne s'oppose pas seulement à son père, il s'engage dans la voie d'une désidéalisation passant par l'attaque des références idéales et esthétiques paternelles. La confiance joue alors un rôle fondamental: prendre confiance en lui en quittant l'identification sur laquelle son jeu s'appuyait, et donc accepter de perdre une série de match avant de gagner différemment, pour lui. Une phrase représente l'importance de la confiance, celle prononcée par son coach Brad Gilbert lorsque, perdu dans sa dépressivité, il lui annonce comme un rituel ou un gimmick: « De bonnes choses vont arriver ».

Il fonde 1'Andre Agassi College Preparatory Academy avant la fin de sa carrière, réalisant qu'il a participé, notamment à l'adolescence, au sabotage de son manque d'éducation : «Je sens le poids de ce manque, le handicap qu'il représente. C'est un crime dont je me suis rendu complice. » (Ibid, p. 421), conclue-t-il en toute lucidité, écartant tout clivage entre agresseur et victime.

\section{Tomber amoureux, un autre usage du corps}

Le sport peut décaler un adolescent de sa violence; cette violence devient une mise en acte encadrée par un dispositif sportif ou thérapeutique symboligène, quand l'environnement le permet. A l'adolescence, les parents orientent alors la vie de leur enfant en évitant les récifs de l'abandon ou de l'emprise. Mais comment ne pas se sentir abandonné lorsqu'on quitte sa famille à treize ans ? Les rationalisations que se racontent les parents et les adolescents n'y font rien, la souffrance et la dépression sont au rendez-vous, troublant l'élaboration du processus, qui semble s'étirer tout au long de la carrière de certains professionnels. B. Borg ne 
s'effondra-t-il pas à vingt-quatre ans, épuisé d'être Ice-Borg, un corps-machine froid et dénué de sensations ? La suite de sa dérive, mêlant addictions diverses et échecs sentimentaux comme professionnels le poussèrent à envisager de vendre un de ses trophées les plus extraordinaire, une des coupes qu'il gagna lors d'un tournoi de Wimbledon. Il fut en son temps considéré comme le meilleur joueur de tennis de tous les temps; le jeu était devenu pour lui une ritournelle obsédante, une plongée progressive dans la dépression post-victoires qu'il n'a pas eue l'occasion de vivre au moment de son adolescence vive. Il est des victoires au goût amer.

Comme le montre le sport à travers l'exemple d'Agassi, un père peut être aussi fou qu'une mère, aussi intolérant à la déception et pathogène qu'une mère insuffisamment bonne. L'idéologie arbitraire inculquée dès la petite enfance évoque une forme d'identification projective. Le fantasme d'introduire de force son propre self dans l'objet tend à obtenir le contrôle réel de l'enfant, la mère comme le père ne pouvant pas tolérer l'accès à l'altérité de son enfant. Une des visées les plus pathologiques de l'identification projective consiste à annuler transitoirement ou durablement la séparation et la différence avec l'objet (Klein, 1952), entraînant une attaque permanente contre l'activité de liaison entre les pensées, qui se transforment en objets bizarres.

«Le centre de gravité de l'expérience identitaire change, de façon sauvage et continuelle à l'adolescence » indique de façon générique D. Meltzer (1967, p. 105). Si on considère le parcours d'Agassi, la potentialité psychotique de l'adolescence y est incarnée via une certaine confusion des désirs, représentant le danger suprême qui menace le moi adolescent : perdre ce qui lui a permis de se construire et se percevoir comme un ensemble insuffisamment cohérent pour assurer son sentiment d'identité et la continuité de son self.

L'intromission des idées paternelles et leur destin psychique chez l'enfant et l'adolescent que fut Agassi n'est pas sans évoquer un brouillage des places et des désirs, entravant le travail de subjectivation adolescent dans le sens d'un vécu de confusion. En suivant le propos de Bion (1962), la confiance de base dans les premiers objets d'amour internalisés s'en trouve attaquée, ce qui a pour effet d'empêcher une prise de conscience des expériences émotionnelles. Celles-ci apparaissent ici comme un des éléments fondamentaux de la trouvaille de la vérité source d'auto-réflexivité et de santé psychique. Le fondement introjectif des identifications perd de sa force, tandis que s'imposent les identifications projectives ou adhésives. Imitation servile et caricature en découlent, au service de l'excès en lieu et place de l'usage de l'imagination. Pour Meltzer (1898), les deux issues d'un ratage du processus adolescent se déclinent de la sorte : les adolescents piégés par l'identification projective et sa 
conséquence, l'emprisonnement claustrophobique, ont tendance à retomber dans une soumission propre à la période de latence ; pour ceux qui obtiennent du succès, l'adolescence serait une problématique interminable, dans le sens d'une installation sans limites dans ce mode de vie et d'être.

Il ajoute que tomber amoureux, au sens élargi, d'une personne ou d'une idée, permet de retrouver le sens de la réalité psychique, de l'individualité, du sentiment d'être un enfant face au mystère et à la beauté du monde. L'aire intermédiaire et la sensibilité poétique sont ici désignées comme les signes d'une vie infantile arrimée à la vie fantasmatique. Tomber amoureux, d'un autre qui puisse comprendre comme une part de soi tout en étant différent, et rester sensible à une « poétique de soi », sans doute sont-ce des éléments qui ont «sauvé » Agassi d'un destin psychopathologique plus radical: avec la fondation de sa fondation « réparatrice » des adolescents en souffrance dans leur environnement, sa rencontre avec Steffi Graf, immense championne de tennis, fut probablement une des issues les plus heureuses qu'il trouva pour rétablir un sentiment d'identité profondément ébranlé, au risque d'un clivage potentiel. La rencontre amoureuse, un autre usage du corps articulant relation d'objet différencié et narcissisme, voici sans doute un remède universel.

\section{$\underline{\text { Bibliographie }}$}

Agassi A., Open, Paris, Plon, 2009.

Bion W. R. (1962), Aux sources de l'expérience, Paris, PUF, 1979.

Freud S. (1905), Trois essais sur la théorie de la sexualité, Paris, Gallimard, 1962.

Gilbert B., Winning Ugly, New York, Touchstone, 1994.

Houssier F., Vlachopoulou X., Bonnichon D., Capart N., Tensions dans la transmission : Sigmund Freud consultant, à partir des lettres inédites adressées à Paul Federn, in Revue Française de Psychanalyse, 2, 2014, à paraître.

Klein M. (1952), Notes sur quelques mécanismes schizoïdes, in M. Klein, P. Heimann, S. Isaacs, J. Riviere (dir.), Développements de la psychanalyse, Paris, PUF, p. 274-300.

Meltzer D., Identification and socialization in adolescents, in Contemporary Psychoanalysis, 3, 1967, p. 96-103.

Meltzer D., Le narcissisme et la violence chez les adolescents, Journal de la psychanalyse de l'Enfant, 7, 1989, p. 226-252. 